\title{
Factores predictores de conductas promotoras de salud en docentes de enfermería de tres universidades de Cali, Colombia
}

\author{
Predictive factors of health promoting behavior in nursing teachers from three universities in Cali, Colombia
}

Zaider Gloria Triviño-Vargas' orcid.org/0000-0001-6968-9334

María Ximena López-Hurtado1* orcid.org/0000-0001-5668-1659

1 Escuela de Enfermería, Universidad del Valle. Santiago de Cali, Colombia

Fecha de recepción: Septiembre 17-2017 Fecha de revisión: Noviembre 10-2017 Fecha de aceptación: Abril 13 - 2018

Triviño-Vargas ZG, López-Hurtado MX, Factores predictores de conductas promotoras de salud en docentes de enfermería de tres universidades de Cali, Colombia. Univ. Salud. 2018;20(2):160-170. DoI: http://dx.doi.org/10.22267/rus.182002.120

\section{Resumen}

Introducción: La promoción de la salud ha sido una preocupación constante en el ser humano, permite comprender comportamientos relacionados con la misma y orienta hacia la generación de conductas promotoras de salud (CPS) que promueven el bienestar personal. Objetivo: Determinar los factores predictores que influyen en las CPS de los docentes según modelo Pender. Materiales y métodos: Diseño descriptivo correlacional, con una muestra representativa de los docentes con contrato vigente durante el estudio. Se aplicaron instrumentos como la escala EVPS II de Pender para medir la variable dependiente CPS, y para las independientes como sociodemográficas, conocimientos sobre promoción de salud, percepción del estado de salud y percepción de autoeficacia. Resultados: Son descriptivos de acuerdo con el coeficiente de variación en cada una de las subdimensiones de las conductas: Crecimiento espiritual, Relaciones interpersonales, Responsabilidad en salud, Nutrición, Manejo del estrés y Actividad física. Los factores predictores resultaron de acuerdo con el modelo de regresión múltiple. Conclusiones: La CPS favorables fueron el crecimiento espiritual, las relaciones interpersonales y la nutrición; los predictores que ejercen efecto sobre las CPS se presentaron en función del escalafón docente, edad, área de desempeño adulto anciano, estrato, ingresos y área de desempeño salud mental y psiquiatría.

Palabras clave: Promoción de la salud; docentes; enfermería (Fuente: DeCS, Bireme).

\begin{abstract}
Introduction: Health promotion has been a constant concern in the human being. It allows to understand behaviors related to it and orientates towards the generation of health promoting behaviors (HPB) that promote personal wellbeing. Objective: To determine the predictor factors influencing the HPB of the teachers according to Pender's health promotion model. Materials and methods: A correlational descriptive design was made with a representative sample of the teachers with current contract during the study. Instruments such as the EVPS II scale of Pender were applied to measure the dependent variable CPS, as well as for the independent ones such as sociodemographic, knowledge on health promotion, perception of health status and perception of self-efficacy. Results: They are descriptive, according to the coefficient of variation in each of the subdimensions of the behaviors: spiritual growth, interpersonal relations, responsibility in health, nutrition, management of stress and physical activity. The predictor factors were based on the multiple regression model. Conclusions: Favorable HPB were spiritual growth, interpersonal relationships and nutrition. Predictors that have effect on HPB were presented on the basis of teacher rankings, age, senior adult performance area, stratum, income and area of performance, mental health and psychiatry.
\end{abstract}

Keywords: Health promotion; faculty; nursing. (Source: DeCS, Bireme). 


\section{Introducción}

La Organización Mundial de la Salud (OMS)(1) promulgó la carta de Ottawa, la cual planteó cinco áreas fundamentales para la promoción de la salud: desarrollo de actitudes personales, el mejoramiento de los entornos o ambientes saludables y la reorientación de los servicios de salud. De acuerdo con lo anterior, es fundamental para la promoción de la salud, el compromiso del docente en términos de conductas y comportamientos salutogénicos; otro aspecto es referido a los entornos y ambientes como la universidad donde permanecen los profesores la mayor parte de su tiempo, y la reorientación de los servicios de salud que coincide con una nueva expectativa, frente a un mayor énfasis hacia la atención primaria en salud y el desarrollo familiar y comunitario en Colombia(2).

Hay un reconocimiento general de la importancia de los docentes en el logro de los objetivos y metas de los procesos educativos que, sin ser el único, es uno de los factores determinantes en las conductas de los jóvenes y sociedad(3,4). Las universidades y sus docentes, además de satisfacer el reto de formar profesionales y ciudadanos cultos y capaces de configurar sociedades solidarias y de progreso, tienen un nuevo reto que afrontar, ser un contexto de convivencia e interrelaciones que facilite comportamientos saludables y que redunde en la calidad de vida, no solo de la comunidad universitaria sino de toda la sociedad en general. Con relación al docente universitario, el inicio de su carrera se caracteriza por ser una etapa llena de tensiones y de situaciones de aprendizaje intenso, donde la filosofía y su concepción de vida debería tener relación directa con su ser, saber y saber hacer, teniendo efectos sobre su comportamiento y estilo de vida(5).

Se considera importante en los docentes, atender de manera satisfactoria las demandas sociales y las de sus estudiantes, lo cual requiere el cuidado de sí como también de su ambiente físico y social. Sin embargo, se conoce muy poco acerca de su calidad de vida general, trabajo o salud. Los profesores universitarios constituyen una población de relevancia e interés para la realización de estudios de bienestar y promoción de su salud, dado su significativo papel como educadores y modelos ejemplares, y también, por el prestigio social y la relevante posición que ocupan en la vida pública(6).

En el contexto de la promoción de la salud, la salud ha sido considerada como un medio para llegar a un fin, como un recurso que permite a las personas el logro de una vida individual, social y económicamente productiva; se trata de un concepto positivo que acentúa los recursos sociales y personales, en este caso del docente( ${ }^{(7)}$.

El modelo de Pender, desarrolla la promoción de la salud como primer enfoque, identificando los factores cognitivos y perceptuales, los cuales pueden ser modificados por las diferentes condiciones personales, interpersonales $y$ situacionales, que dan salida a resultados y respuestas de la conducta de las personas, constituyéndose en determinantes del estilo de vida promotor de la salud (EVPS), calificado a través de una escala. El objetivo del presente estudio fue determinar los factores predictores de conductas promotoras de salud en docentes de enfermería de tres universidades de Cali, describiendo el perfil de las conductas favorables y menos favorables.

\section{Materiales y métodos}

Estudio descriptivo, transversal y correlacional, con un universo de 170 docentes enfermeros de tres universidades de Cali, con contratación vigente. La muestra, se realizó por convocatoria, en la cual 126 docentes accedieron a participar espontánea y voluntariamente. Esta muestra correspondió al 74\% del universo. Los criterios de inclusión fueron docentes con relación laboral por contrato o por nombramiento, que trabajaran en alguna de las tres universidades de Cali durante el tiempo de aplicación de la encuesta estructurada y que dieran su consentimiento informado. La variable dependiente fue la Conducta Promotora de Salud (CPS), la cual fue medida a través de la Escala Estilo de Vida Promotor de Salud (EVPS II) de Pender, Walker y Schrist(8). Las variables 
independientes fueron: sociodemográficas, conocimientos sobre promoción de salud; variables psicológicas: percepción del estado de salud y percepción de autoeficacia; biológicas: peso, edad y género; socioculturales: etnia, estado civil, estrato social, procedencia, tenencia de hijos, nivel de formación y salario. La EVPS II de Pender, Walker y Scherist presenta una confiabilidad por Cronbach de 0,93, su validez fue reportada por análisis factorial de componentes principales y ha sido utilizada por Triviño, Merino y Stiepovich en el contexto colombiano en mujeres caleñas ${ }^{(9)}$. La escala se estructura por 52 ítems con opciones de respuesta tipo Likert donde nunca es igual a 1 , algunas veces corresponde a 2 , frecuentemente es 3 y siempre 4 , presenta 6 subdimensiones: crecimiento espiritual, manejo del estrés, actividad física, relaciones interpersonales, nutrición y responsabilidad en salud.

Otros instrumentos utilizados fueron la Escala de Percepción de Autoeficacia de Blaber y Schwarzer(10), que reporta una confiabilidad por Cronbach de 0,75 a 0,91 y validez de constructo; la Escala de Estado de Salud Percibido de Recker y Wong(11), con una confiabilidad de 0,73 y el test sobre conocimientos de promoción de la salud. Los cuestionarios sobre características sociodemográficas y sobre conocimientos de promoción de la salud fueron elaborados por las autoras.

Para el análisis de datos descriptivos de las variables, se reconoce que la escala es numérica lo que permite utilizar medidas como promedio, desviación estándar y variabilidad, donde se presentan las diferentes subdimensiones y se comparan entre ellas. Para el análisis del modelo, se verificó normalización a través de la prueba de Kolmogorov-Smirnov (K-S), encontrándose normalidad en todas las variables excepto en la de conocimientos. El análisis multivariado se realizó a través de regresión múltiple, para determinar los factores que predicen el estilo promotor de salud con un nivel de confianza de 0,05. Se discernió mediante análisis de regresión jerárquica, para observar los factores que incidieron porcentualmente en la conducta promotora de salud, esto permitió visualizar cuales fueron los más importantes predictores entre los factores que influyeron en la variación de la conducta promotora de salud. Se utilizó el programa estadístico SPSS ${ }^{\circledR}$ versión 17.

\section{Consideraciones éticas}

El estudio está catalogado como una investigación con riesgo mínimo de acuerdo con la Resolución 08430 de 1993 y además contó con los respectivos avales del Comité de Ética de las universidades participantes, según Acta de aprobación No. 011-011 del 19 de junio de 2011 de la Universidad del Valle, Acta No. 24 del 7 de marzo de 2012 de la Universidad Libre Seccional Cali y Acta $\mathrm{N}^{\circ} 03$ del 7 de marzo de 2012 de la Universidad Santiago de Cali.

\section{Resultados}

El 94\% de los participantes fueron mujeres, con un peso promedio de $62,4 \mathrm{~kg}$ y edad promedio de 48,9 años siendo la edad mínima 24 y la máxima 69 años. El 63,5\% de los participantes fue de etnia mestiza, el resto se distribuyó entre la etnia blanca y negra; la mayoría (54\%) no viven en pareja, el 69\% reportó tener hijos y el 76\% pertenece al estrato 4, 5 o 6; con respecto a la procedencia el 50,8\% proviene del Valle del Cauca, el 33,3\% proviene del Cauca, Nariño y Eje cafetero y el $15,9 \%$ proviene de otras regiones.

En cuanto a conocimientos sobre promoción de la salud el puntaje máximo posible eran 15 puntos, 12 (9,5\%) participantes lo obtuvieron, el $92,5 \%$ demostró tener conocimientos sobre promoción de la salud considerando que 9 puntos o más son aprobatorios. Frente a la percepción del estado de salud, el puntaje varió entre 15 y 89 puntos (máximo teórico de 112 puntos) y el promedio fue 66,5 puntos; en cuanto a la autoeficacia la variación estuvo entre 10 y 40 puntos (máximo teórico de 40 puntos) y un promedio de 33,5 puntos.

La Tabla 1 presenta el análisis descriptivo de los puntajes alcanzados por los docentes en la CPS según subescalas de la Escala Estilo de Vida Promotor de Salud (EVPS II) de Pender. Configurándose de forma cuantitativa; el puntaje total podría variar entre los valores de 52 a 208 . 
Las columnas tituladas Mínimo y Máximo muestran los valores reales, obtenidos en cada subdimensión. La distribución real de los puntajes en este estudio fue de 101 puntos el mínimo y 197 el máximo, con un promedio de 152,36; en esta distribución la desviación estándar fue de 18,85 y un coeficiente de variación de 12,37\%.

El coeficiente de variación permite describir mejor el comportamiento diverso de los participantes, a pesar de ser todos profesionales y todos docentes aún de la misma disciplina de enfermería. Las subdimensiones de crecimiento espiritual, relaciones interpersonales y nutrición presentaron los menores puntajes de variación, así: 10,74\%, 12,89\% y 13,85\% respectivamente.

El análisis según el coeficiente de variación corroboró que las subdimensiones de actividad física, manejo del estrés y responsabilidad en salud, tuvieron los mayores coeficientes de variación así: $30,32 \%, \quad 19,71 \%$ y 18,31\% respectivamente.

Tabla 1. Distribución según subdimensiones de escala Estilo de Vida Promotor de Salud II de Pender en docentes de Enfermería de tres universidades de Cali

\begin{tabular}{|c|c|c|c|c|c|c|}
\hline Subdimensiones & $\mathbf{N}$ & Mínimo & Máximo & Media & $\begin{array}{c}\text { Desviación } \\
\text { típica }\end{array}$ & $\begin{array}{l}\text { Coeficiente de } \\
\text { variación }\end{array}$ \\
\hline Responsabilidad en salud & 126 & 14 & 36 & 25,54 & 4,67 & 18,31 \\
\hline Crecimiento espiritual & 126 & 22 & 36 & 31,19 & 3,35 & 10,74 \\
\hline Manejo del estrés & 126 & 10 & 55 & 21,21 & 5,11 & 19,71 \\
\hline Actividad física & 126 & 8,0 & 32 & 20,15 & 6,13 & 30,32 \\
\hline Relaciones interpersonales & 124 & 17 & 56 & 29,06 & 4,46 & 12,89 \\
\hline Nutrición & 125 & 18 & 34 & 25,64 & 3,56 & 13,85 \\
\hline Total CPS & 126 & 101 & 197 & 152,36 & 18,85 & 12,37 \\
\hline
\end{tabular}

Fuente: Factores predictores de conductas promotoras de salud en docentes de enfermería de tres universidades de Cali, 2014.

La Figura 1 presenta las subdimensiones de la escala Estilo de Vida Promotor de Salud (EVPS II) de Pender, donde se observó que la conducta con más alto puntaje promedio fue la de crecimiento espiritual, seguida de las conductas de relaciones interpersonales y nutrición; las conductas con menores promedios observados fueron actividad física, manejo del estrés y responsabilidad en salud. Al estimar el modelo de regresión se generó el modelo óptimo seleccionando las variables independientes, es decir aquellas que tuvieron la más alta correlación observada.
La Tabla 2 presenta como en el análisis multivariado, los predictores que mayor fuerza ejercieron sobre las conductas promotoras de salud fueron las variables: escalafón, la edad, trabajar en el área adulto anciano, el estrato socioeconómico, los ingresos y trabajar en el área de salud mental; alcanzaron el 34\% de la explicación en la variabilidad de la conducta promotora de salud en los docentes. Se observó que entre las variables biológicas, solo ingresó en el modelo la variable edad.

Tabla 2. Análisis multivariado de seis predictores de la variable dependiente Conducta promotora de salud

\begin{tabular}{|c|c|c|c|c|c|}
\hline Predictores (6) & SS Total & SS Modelo & gl & $\mathbf{R}^{2}$ & $\mathbf{P}$ \\
\hline $\begin{array}{l}\text { Escalafón + Edad + Área adulto anciano + Estrato + Ingresos + Área de } \\
\text { salud mental }\end{array}$ & 21652 & 9362 & 6 & 34,0 & 0,001 \\
\hline Escalafón + Edad + Área adulto anciano + Estrato + Ingresos + Etnia & 21652 & 8664 & 6 & 30,3 & 0,003 \\
\hline Escalafón + Edad + Área adulto anciano + Estrato + Ingresos + Contrato & 21652 & 8372 & 6 & 28,7 & 0,004 \\
\hline
\end{tabular}




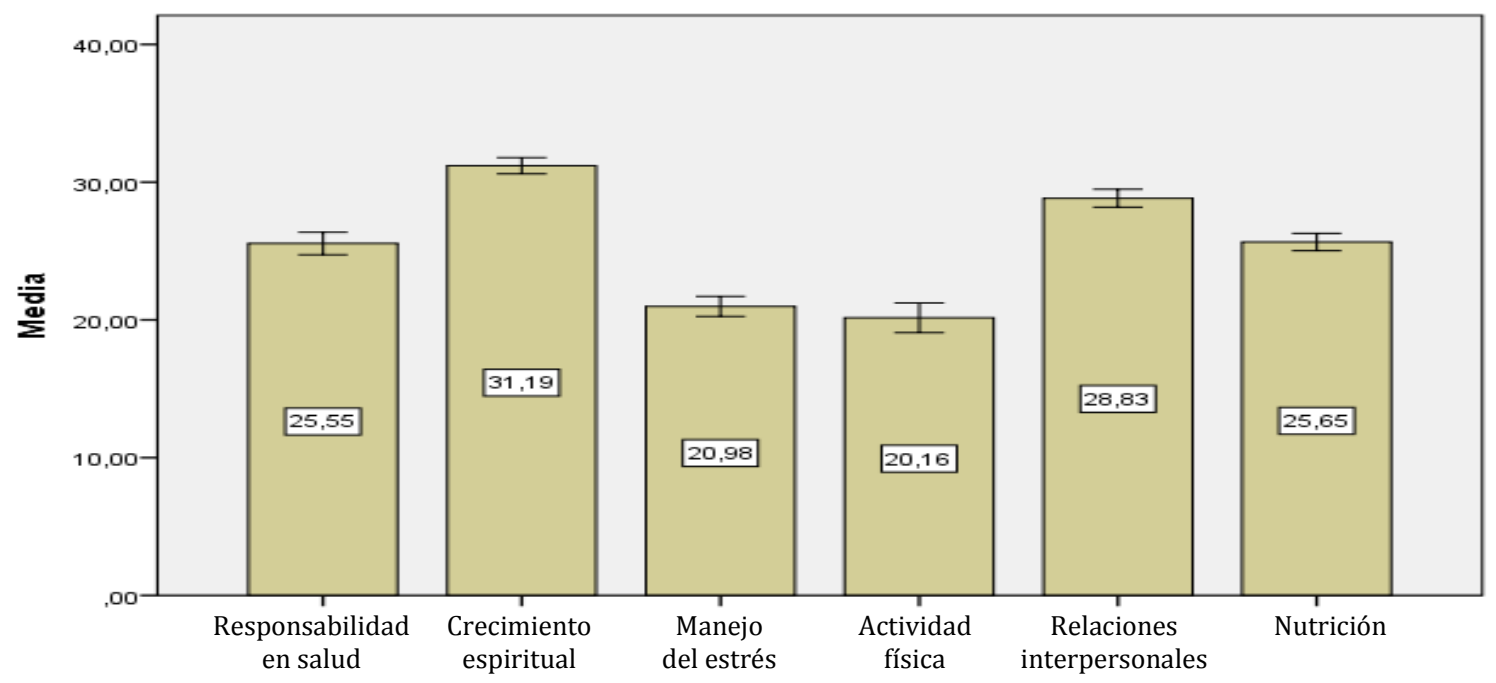

Figura 1. Descriptivo de subdimensiones de la escala Estilo de Vida Promotor de Salud II de Pender en docentes de Enfermería

Los resultados aquí relacionados se refieren a la construcción del modelo. Las variables que no aparecen, aunque fueron analizadas, no tuvieron peso o correlación con la conducta promotora de salud.

\section{Discusión}

Con respecto al perfil de los docentes de enfermería de las tres universidades de Cali, la mayor proporción fue de género femenino, este hallazgo se considera lo esperado debido a que tradicionalmente esta profesión ha sido ejercida por mujeres, dato similar al reportado por Petri et al,.(12); a pesar de que actualmente por las transformaciones socioculturales y políticas la participación del género masculino aumenta en la ciencia y disciplina de enfermería(13); la mujer también ha asumido socioculturalmente el rol del cuidado de la familia(14).

La edad promedio fue de 48,9 años correspondiente a adulto maduro según clasificación de la OMS(15), en mayor proporción fueron personas casadas, pero si al sumar las solteras(os), separadas(os) y viudas(os) se observa que es mayor el número de docentes sin unión marital, este hallazgo puede dar cuenta sobre la necesidad de asumir los roles como gestor del hogar y profesor de enfermería, donde ejercer la docencia se vuelve un desafío(16). Los participantes se atribuyen de etnia mestiza y las universidades cuentan con docentes de procedencia del Valle del Cauca en mayor proporción, observándose también docentes de otras regiones, lo cual puede considerarse un efecto positivo frente a la diversidad y experiencias significativas que aportan cada una frente a los procesos de enseñanza aprendizaje para los estudiantes.

Los docentes en su mayoría viven en estrato socioeconómico $4 \mathrm{y} 5$. El promedio salarial estuvo en $\$ 2.550 .000$, este reporte de salario muestra que es superior al promedio nacional referenciado por la Asociación Nacional de Enfermeras de Colombia (ANEC)(17), aunque los docentes trabajan en otras instituciones de nivel superior para proveerse un mayor ingreso por mes, vale la pena mencionar que el menor ingreso fue de $\$ 2.000 .000$ y el más alto de $\$ 9.000 .000$ generando una gran dispersión; esto puede deberse a que una de las universidades participantes es de carácter privado y no aplica el escalafón docente, además a los tipos de contratación y antigüedad en la labor docente(18); con relación a esta última, en promedio fue reportada en 9,6 años lo que favorece un mejor proceso en la enseñanza disciplinar y la pedagogía como formas y métodos más estructurados $y$ fortalecidos de enseñanza- 
aprendizaje. En las universidades participantes en el presente estudio, predominó la contratación de profesores por hora cátedra $(61,1 \%)$, hallazgo que coincide con lo reportado por Cuninghame ${ }^{19)}$ citado por Tamez y Pérez que plantea que alrededor del $60 \%$ del total de trabajadores dedicados a la educación están vinculados por este tipo de contratación.

En cuanto a las áreas de desempeño se evidenció que los programas académicos están estructurados por ciclos vitales: niño, madre, adulto y anciano; en este marco las docentes laboran en más de un área de desempeño en la misma o en otras universidades. Este hallazgo se somete a discusión frente a la necesidad de los docentes de incrementar sus ingresos aún a pesar de su desgaste laboral(20).

El mayor nivel de formación alcanzado fue doctorado, sin embargo la evidencia mostró mayor proporción de formación en maestría; coherente con los requisitos actuales de convocatoria docente en las tres universidades colombianas participantes, de acuerdo con los reglamentos y perfiles docentes requeridos.

Se observó que la formación doctoral en los docentes es baja, situación que puede asociarse al factor económico y a la vinculación hora cátedra, con términos diferentes a los establecidos para los docentes de planta, situación que dificulta el ascenso profesoral en la carrera docente, por limitaciones en las becas y estímulos.

El $64,3 \%$ de los docentes no ha accedido a escalafonamiento, dado que está supeditado a la cualificación de los mismos, relacionado con los méritos, la producción intelectual, el tiempo de vinculación, que son variables diferenciales para cada persona dentro de su carrera docente; situación que se considera tiene efectos sobre el ascenso profesoral, y que apoyan procesos de calidad para acreditación de las universidades(21).

Las docentes de enfermería reportaron bajo consumo de bebidas alcohólicas, el 53,8\% no bebe nunca, y bajo hábito de consumo de tabaco siendo que el 94,4\% no fuman, situación favorable para una CPS de los mismos; este dato contrasta con los resultados encontrados en docentes de la Universidad de Costa Rica, donde el $10 \%$ fuma cigarrillos diariamente, el $16 \%$ consume alcohol los fines de semana y casi el $20 \%$ una vez a la semana(22).

\section{Perfil de las conductas promotoras de salud en los docentes}

\section{Espiritualidad}

Con relación a la conducta de crecimiento espiritual los docentes presentan una alta espiritualidad puesto que sienten que su vida tiene un propósito, creen en un ser superior sin importar la religiosidad; este hallazgo es consistente con lo reportado por Pérez(23) quien describe que la espiritualidad implica la búsqueda de un significado y el descubrimiento personal, especialmente a través del trabajo, considerado como un medio de expresión de las necesidades internas del individuo, convirtiéndolo en una forma de expresar la espiritualidad. Esta conducta podría ser producto de los procesos personales vividos por los docentes que les permite mayor autodeterminación y trascendencia. En otro sentido, el crecimiento y desarrollo espiritual a través del trabajo, en este caso de la docencia, es un proceso continuo de crecimiento y desarrollo personal, que se manifiesta en el nivel alcanzado de integración, significado y trascendencia, que genera en los docentes fuerza y una actitud positiva hacia el trabajo(18).

\section{Relaciones interpersonales}

Las relaciones interpersonales en los docentes se consideraron favorables, situación que podría estar influenciada por la constitución de redes de apoyo y trabajo en equipo de las docentes que les permite la capacidad de conciliar las múltiples actividades que genera la docencia. Estos resultados son consistentes con lo informado por Merighi et al.(16), estudios reportan que el $43 \%$ de los docentes pertenece a algún grupo social, político o religioso(18); se discute que posiblemente este hallazgo neutralice las altas cargas de estrés encontradas. 


\section{Nutrición}

La conducta nutrición alcanzó un promedio con tendencia moderadamente alta, dado que su coeficiente de variación fue de 13,85 , que podría estar sugiriendo el conocimiento que tienen los docentes sobre alimentación saludable, su requerimiento, cantidad y calidad en el consumo de la misma; este comportamiento es el esperado teniendo en cuenta que los conocimientos y prácticas de los profesionales de enfermería sobre alimentación saludable son suficientes y pueden representar un factor protector para su salud. Sin embargo, es pertinente considerar las pocas ofertas de alimentos nutritivos y saludables en las cafeterías de las instituciones educativas y los factores económicos, son determinantes para el consumo y calidad de la alimentación según lo expresado por la OMS(24). Estas conductas nutricionales adecuadas coinciden con lo reportado por Sandoval en la encuesta realizada a docentes, con respecto a la integridad e inclusión en el consumo diario de frutas en el $69 \%$ y verduras en el $66 \%$ de los docentes(22).

\section{Responsabilidad en salud}

En orden descendente se ubicó responsabilidad en salud, la cual presentó un coeficiente de variación alto. Este hecho resalta quizás la incoherencia en la responsabilidad sobre su salud siendo agentes de la misma. Podría indicar también que la relación laboral de las cargas que asume en sus currículos no le permite mayor conciencia y práctica de sus responsabilidades biopsicosociales vistos como un todo puesto que no se afecta, solamente la salud física sino también las otras esferas(25). Aquí también puede sobresalir la situación que se encontró en este estudio, donde los docentes trabajan dobles jornadas y en diferentes universidades con el fin de mejorar sus ingresos(26); aunque esta situación no se dio en el caso de una de las universidades que fue la pública. Aquí se somete a discusión que el docente, sopena de su carga laboral, siempre debe tener claridad sobre la importancia de hacer promoción de su salud.

\section{Manejo del estrés}

La conducta sobre manejo del estrés ocupó el penúltimo lugar entre las conductas con coeficiente de variación alto, lo cual podría ser sugestivo de las dificultades que tienen los docentes relacionadas con el compromiso del aprendizaje más que la enseñanza, pues desde la perspectiva pedagógica se cree que tiene mayor trascendencia el aprendizaje, esto es que los medios utilizados por los docentes sean captados o aprehendidos por los estudiantes; esto muestra concordancia con lo reportado por Olmedo et $a l, .(27)$. La carga académica en los docentes juega un papel importante en la presencia de estrés, así también la lucha por su posicionamiento en el escalafonamiento en su carrera docente y en su reconocimiento. Otro factor que debe destacarse está relacionado con la situación laboral de trabajar en más de una institución de educación superior.

Así también otros estudios publicados informan que el estrés laboral se incrementa de acuerdo con el nivel de productividad académica del profesor evaluado a través de los programas de estímulos, lo que podría sugerir que la carga emocional y el desgaste físico que conlleva la necesidad de incrementar la productividad, puede ser un factor coadyuvante o detonante de estrés(28-31).

Un estudio que merece mención es el reportado por Sánchez y Clavería(32), donde se revisó y valoró el estrés laboral en docentes de la Universidad de Lleida, España, quienes mostraron su percepción personal de malestar, con un síndrome de Burnout moderado, superior en mujeres, profesores de menor escalafón y menor antigüedad, quienes suelen tener más problemas con su promoción personal y sus salarios.

\section{Actividad física}

La última conducta referida a mayor coeficiente de variación y dispersión fue la actividad física, en la cual se reconoce empíricamente que los docentes no tienen incorporada la conducta de actividad física, podría estar influenciada por los avances tecnológicos como el uso de vehículos, ascensores, entre otros. Posiblemente la rutina de trabajo, hace que perpetúe esta ausencia de actividad física. Los docentes deben crear conciencia sobre los efectos positivos de la 
actividad física en su salud(3), otros estudios coinciden con este reporte de falta de actividad física en otras profesiones y poblaciones(33). El hallazgo de baja actividad física, coincide con datos reportados en el estudio en docentes universitarias de enfermería en Ecuador, donde el $83 \%$ de la población no cumple con las recomendaciones de práctica de actividad física de la OMS(34). Este hallazgo dificulta las conductas promotoras de salud en la generación de estilos de vida y trabajo saludables, hecho que debe generar compromiso con la implementación de instituciones con ambientes saludables de trabajo, como lo contempla la carta de Ottawa en cuanto a los cinco determinantes para el mejoramiento de los entornos o ambientes saludables.

Una de las proposiciones teóricas del modelo de promoción de la salud de Pender establece que las barreras percibidas pueden impedir el compromiso con la acción, y que las personas se comprometen a involucrarse en conductas de las cuales ellas anticipan beneficios personalmente valorados. La percepción de autoeficacia o competencia para ejecutar una conducta incrementa la posibilidad del compromiso con la acción(35). Posiblemente los docentes no anticipan beneficios personales, no se perciban competentes o autoeficaces y presentan barreras percibidas para ejecutar la conducta de actividad física. Puede también considerarse que las múltiples actividades de lo cotidiano lleven a los docentes a dejar en segundo plano el cuidado de sí mismos, la rutina de vida, la gerencia de la casa, el cuidado de los hijos, estudio, preparación y ejecución de actividades de enseñanza y en general la dinámica laboral a que están sometidos no les permite disponer de tiempo para el autocuidado(16).

Con respecto al análisis multivariado, los factores predictores que influyen en la variabilidad de la CPS en los docentes, se evidenciaron el escalafón, la edad, pertenecer al área adulto anciano, de salud mental, el estrato, e ingresos.

El escalafón, es una clasificación del profesorado según su experiencia docente profesional, académica e investigativa, académico- administrativa; diseñado para estimular la excelencia académica de los docentes y por ende de la institución universitaria(36); estimula la vinculación, permanencia y promoción del profesorado; y establece los grados mínimos de calificación para pasar de una categoría a otra, debe existir una adecuada correspondencia entre los ingresos de un profesor y su categoría en el escalafón docente; el cual representa puntos salariales $(37,38)$. El escalafón se muestra teniendo efectos sobre el mejoramiento de la docencia, la pedagogía de la enseñanza, y a su vez de una alta producción de conocimientos; estos efectos permiten al docente acceder a mejor remuneración salarial que favorece el acceso a los componentes y condicionantes del bienestar, entre otros la alimentación, educación, salud, que benefician conductas promotoras de salud. Se somete a discusión el hecho de que el escalafón en contraposición, la empírica ha demostrado relación con el mayor esfuerzo y proceso de desgate- reproducción, en tiempo, en producción intelectual para el docente, lo cual podría ser inverso sobre la conducta promotora de salud; como ha sido manifestado por algunos autores que lo relacionan con síndrome de Burnout(39-41).

Otro factor que entró como predictor de la CPS, fue la edad que coincide con el adulto maduro. Desde la perspectiva de las autoras del estudio, se considera que en este ciclo vital el docente tiene una mejor relación construida, mayor experiencia, autoeficacia y vivencias que lo facultan en procesos pedagógico más acabados, elaborados y experienciales; de esta forma el profesor tiene mayor conciencia en la toma de decisiones y del reconocimiento de lo que está bien o no. De otra parte, también se considera que está influenciado por la experiencia laboral acumulada y no necesariamente por la edad.

También entró como predictor en este estudio, el desempeño en el área de cuidado del adulto y anciano, lo cual demuestra la relación de haber vivido experiencias que le configuran la idea de que si se establecen conductas favorables a menor edad, el futuro saludable está centrado en promover hábitos saludables $\mathrm{y}$ prevenir situaciones que generen procesos mórbidos. El docente tiene mayor claridad $y$ nivel de 
conciencia de que si se adoptan CPS a menor edad se favorecen procesos de vejez salutogénicos. En el modelo de promoción de la salud de Pender, se describe que el estilo de vida se verá afectado por la etapa específica del ciclo vital en la cual se encuentre la persona ${ }^{(42)}$.

Frente al estrato socioeconómico e ingresos, son variables relevantes a considerar en la medida en que están asociadas a situaciones que permiten a las personas tener capacidades y recursos para lograr llevar a la práctica la predisposición existente de determinadas conductas, positivas o negativas(43), en este caso la idea es que la variable ingresos facilite mejores condiciones de vida en los docentes.

El último predictor identificado fue desempeñarse como docente en el área de cuidado de la salud mental. La práctica de la enfermería en salud mental exige un trabajo personal en la propia salud mental, estrategias de afrontamiento saludables de la enfermedad, de las dificultades o de las incapacidades ${ }^{(44) . ~ E n ~}$ este sentido, este predictor puede estar anunciando que la experiencia que acumula el docente en el área de salud mental le da herramientas, habilidades y conceptualizaciones relacionadas con la promoción de la salud mental; así, el docente construye en su propio contexto de enseñanza-aprendizaje, estrategias de afrontamiento personal para transferirlas a sus educandos, de manera que con su aplicación logren superar barreras de ansiedad, estrés, temores y formar estructuras de salud mental más fuertes. Dichas estructuras fuertes se convierten en un factor predictor de salud, en este caso de la salud mental.

\section{Conclusiones}

Las conductas promotoras de salud favorables en este grupo de estudio fueron el crecimiento espiritual, las relaciones interpersonales y la nutrición, debe considerarse el hecho de que la conducta con mayor promedio fue crecimiento espiritual. Se requiere de parte de las universidades participantes, desarrollar acciones positivas para conservar $y$ fomentar estas conductas. Asimismo, establecer estrategias para fortalecer las conductas menos favorables que corresponden a responsabilidad en salud, manejo del estrés y actividad física.

Los predictores de CPS en los docentes estudiados, con una variabilidad del $34 \%$, fueron el escalafón docente, la edad, la docencia en las áreas de adulto anciano y salud mental, el estrato socioeconómico, los ingresos; constituyéndose en factores clave en la promoción de su salud y cuidado personal.

El escalafón permite mejorar la calidad de vida, porque se accede a otros determinantes en la vida y desarrollo de las personas; la edad se relaciona con los procesos de productividad, fuerza y madurez de los procesos cognitivos y experiencia e idoneidad; la docencia en el área adulto anciano favorece la reflexión y conjuga la experiencia acumulada para el desarrollo de sus prácticas saludables; tanto el estrato como los ingresos hacen sinergia proporcionando una mayor estabilidad y tranquilidad en la vida de los docentes. Finalmente, la docencia en el área de salud mental mejora la situación de equilibrio y armonía para el proceso enseñanza-aprendizaje y en su esfera personal.

Los resultados de esta investigación permiten a las instituciones de educación superior disponer de información útil para la construcción y mejoramiento de políticas de gestión del recurso humano docente, que propenda por su salud y bienestar y el fortalecimiento de estándares de acreditación en la calidad de la educación.

Limitaciones y fortalezas. No se logró un mayor número de docentes participantes en la convocatoria para aplicación de los instrumentos, por la dinámica de sus diferentes actividades académicas y diferencias de horarios.

Conflicto de intereses. Ninguno declarado por las autoras.

\section{Referencias}

1. Organización Mundial de la Salud. Carta de Ottawa para la Promoción de la Salud. Primera Conferencia Internacional sobre Promoción de la Salud [Internet]. Ginebra: OMS; 1986. [consultado 2014 Abril 20]. 
Disponible

en: http://www1.paho.org/spanish/HPP/OttawaCharterS p.pdf.

2. Vargas Porras P, Trujillo González S, Muñoz Sánchez A. Desarrollo conceptual de la promoción de la salud en los lugares de trabajo. Enfermería Glob [Internet]. 2010;(20):1-8. Disponible en: http://scielo.isciii.es/pdf/eg/n20/reflexiones1.pdf

3. Zabalza MÁ. Ser profesor universitario hoy. La cuestión Univ [Internet]. 2009;5(5):69-81. Disponible en: http://unmotivo.com/lcu/grafica/articulos/imgs_bolet in_5/pdfs/LCU5-7.pdf

4. Cortés Marín EA. El nuevo rol del docente universitario. Rev CES Med Vet y Zootec. 2007;2:89-99.

5. Hué García C. Bienestar docente y pensamiento emocional. Rev Fuentes. 2012;12:47-68.

6. Herranz Bellido J, Reig Ferrer A (dir), Cabreo García J (dir). La calidad de vida, el trabajo y la salud de los profesores universitarios [tesis doctoral en Internet]. Alicante: Universidad de Alicante; 2004. [citado 20 de octubre de 2016]. Disponible en https://www.researchgate.net/profile/Jesus_HerranzBellido/publication/39436961_La_calidad_de_vida_el_t rabajo_y_la_salud_de_los_profesores_universitarios/lin ks/553946c40cf226723aba1640.pdf

7. Muñoz Sánchez AI, Castro Silva E. De la promoción de la salud a los ambientes de trabajo saludables. Salud los Trab [Internet]. 2010;18(2):141-152. Disponible en: http://dialnet.unirioja.es/descarga/articulo/3415513. pdf

8. Walker SN, Kerr MJ, Pender NJ, Sechrist KR. A spanish language version of the health-promoting lifestyle profile. Nursing Research. 1990 Jan 1;39(5):268-273.

9. Triviño Z, Stiepovich J, Merino JM. Factores predictores de conductas promotoras de salud en mujeres peri post-menopáusicas de Cali, Colombia. Colomb Med. 2007;38(4):395-407.

10. Schwarzer R, Jerusalem M. General self-efficacy scale (GSE). Berlín: Freie Universität Berlín; 2013. 35-7. Disponible en: http:// userpage.fuberlin.de/\%7Ehealth/spanscal.htm

11. Reker GT, Wong PTP. Psychological and physical wellbeing in the elderly: The perceived well-being scale (PWB) [Internet]. Vol. 3, Canadian Journal on Aging / La Revue canadienne du vieillissement. 1984. p. 23-32. Disponible

en: http://www.journals.cambridge.org/abstract_S071498 0800006437

12. Petri Tavares J, Colomé Beck CL, Bosi de Souza Magnano TS, Ruviaro Zanini R, Lautert L. Disturbios psíquicos menores en enfermeros docentes universitarios. Rev Latino-Am Enferm. 2012;20(1):1-8.

13. Osses-Paredes C, Valenzuela Suazo S, Sanhueza Alvarado O. Hombres en la enfermería profesional. Enfermería Glob. 2010;(18):1-7.

14. Mahecha Angulo M, León Espinosa E. Condiciones salud - trabajo de docentes profesionales de enfermería vinculados a programas de formación para auxiliares de enfermería. Tunja. Docencia Univ [Internet]. 2014;35(Julio):148-59. Disponible en: http://ezproxy.unbosque.edu.co:2048/login?url=http:/ /search.proquest.com.ezproxy.unbosque.edu.co/docvie $\mathrm{w} / 1664837270$ ?accountid $=41311$

15. Organización Mundial de la Salud. Envejecimiento [Internet]. 2017 [citado 2017 Agosto 6]. Disponible en: http://www.who.int/topics/ageing/es/

16. Barbosa Merighi MA, Pinto de Jesus MC, Ribeiro de Fonseca Domingos S, Deíse M de O, Campos Pavan Baptista P. Ser docente de enfermería, mujer y madre: revelando la vivencia bajo la perspectiva de la fenomenología social. Rev Lat Am Enfermagem. 2011;19(1):1-8.

17. Prada-Moreno C. Asociación Nacional de Enfermeras de Colombia. Base de datos del RUN aporta indicadores socio laborales de profesionales de enfermería. Rev Anec [Internet]. 2014 Jul [citado 2017 Dic 7]; 78:44- 5. Disponible en: http://www.anec.org.co/revista78/ revista-78.pdf

18. Cuenca, R., Fabara Garzón, E., Kohen, J., Parra Garrido, M., Rodríguez Guzmán, L y Tomasina F. Condiciones de trabajo y salud docente. Estudios de casos en argentina, Chile, Ecuador, México, Perú y Uruguay. [Internet]. Unesco. 2005. 206 p. Disponible en: http://www.facso.uchile.cl/psicologia/epe/_document os/salud_docente/unesco_condiciones_trabajo_salud_d ocente.pdf\%0Ahttp://unesdoc.unesco.org/images/001 4/001425/142551s.pdf

19. Cuninghame P. "EduFactory": precarización de la producción del conocimiento y alternativas. Bajo el Volcán. 2008;7:11-24.

20. Caballero Dominguez C, González Gutiérrez O, Mercado Anguila D, Llanos Barrios C, Bermejo Barrero Y, Vergel Medrano C. Prevalencia del sindrome del burnout y su correlación con factores psicosociales en docentes de una institucion universitaria privada de la ciudad de Barranquilla. Rev Psicogente. 2009;12(21):142-57.

21. Arias Galicia FA, González Zermeño ME. Estrés, agotamiento profesional (burnout) y salud en profesores de acuerdo a su tipo de contrato. Cienc Trab. [Internet]. 2009 jul-sep; 11 (33):172-176. Disponible en:

https://scholar.google.es/scholar?hl=es\&as_sdt=0\%2C $5 \& \mathrm{q}=$ Estr $\% \mathrm{C} 3 \% \mathrm{~A} 9 \mathrm{~s} \% 2 \mathrm{C}+$ Agotamiento+Profesional $\%$ 28burnout $\% 29+y+$ Salud+en+Profesores+de+acuerdo+ $\mathrm{a}+\mathrm{su}+$ tipo+de+contrato\&btnG=

22. Sandoval I. Resultados de la encuesta a docentes universitarios. 2006 [Internet]. San José: Universidad de Costa Rica, Escuela de Estadística; 2007 [citado 23 Nov 2017]. Disponible en: http://www.ucr.ac.cr/medios/documentos/Docentes_ 2006_Informe_Final.pdf

23. Pérez Santiago JA. Estudio exploratorio sobre el tema de la espiritualidad en el ambiente laboral. An Psicol. 2007;23(1):137-46.

24. Organización Mundial de la Salud. Entornos laborales saludables: fundamentos y modelo de la OMS. Contextualización, práctica y literatura de apoyo [Internet]. 2010 [consultado 2013 abril 28]. Disponible en:

http://www.who.int/occupational_health/evelyn_hwp_ spanish.pdf 
25. Lozada M MA. La docencia: ¿un riesgo para la salud? Avances. 2005;XXIII(1):18-30.

26. Tamez González S. Salud y malestar docente: un análisis crítico. 2008;1-10.

27. Olmedo-Buenrostro BA, Delgado-Enciso I, López-Lavín M, Yáñez-Velazco JC, Mora-Brambila AB, VelascoRodríguez $\mathrm{R}$, et al. Perfil de salud en profesores universitarios y su productividad. Rev Cuba Investig Biomed. 2013;32(2):130-8.

28. Weber A, Lederer P. Morbidity and early retirement among teachers at vocational chools. Versicherungsmedizin. 2006 mar 1;58(1):22-8.

29. Bauer J, Stamm A, Virnich K, Wissing K, Muller U. Correlation between burnout syndrome and psychological and psychosomatic symptoms among teachers. Int Arc Occup Environ Health. 2006 mar;79(3):199-204.

30. Pithers RT, Fogarty GJ. Occupational stress among vocational teachers. $\mathrm{Br} \mathrm{J}$ Educ Psychol [Internet]. 1995;65(1):3-14. Disponible en: http://doi.wiley.com/10.1111/j.20448279.1995.tb01127.x

31. Friedman IA. Burnout in teachers: Shattered dreams of impeccable professional performance. J Clin Psychol. 2000;56(5):595-606.

32. Sánchez-Fernández M, Clavería-Señis M. Profesorado universitario: estres laboral. Factor de riesgo de salud. Enfermería Glob Rev electrónica Semest enfermería [Internet]. 2005;(6):12-6. Disponible en: http://dialnet.unirioja.es/servlet/articulo?codigo $=121$ 1085\&info=resumen\&idioma=ENG

33. Roldán Aguilar EE, Lopera Zapata MH, Londoño Giraldo FJ, Cardeño Tejada JL, Zapata Vidales SA. Análisis descriptivo de las variables: nivel de actividad física, depresión y riesgos cardiovasculares en empleados y docentes de una institución universitaria en Medellín (Colombia). Apunt Med L'Esport [Internet]. 2008;158:55-61. Disponible en: http://appswl.elsevier.es/watermark/ctl_servlet?_f=10 \&pident_articulo $=13125202 \&$ pident_usuario $=0 \&$ pcont actid $=$ \&pident_revista $=277 \&$ ty $=97 \&$ accion $=$ L\&origen $=$ bronco\&web $=w w w$.apunts.org\&lan $=$ es \&fichero $=277 \mathrm{v} 4$ 3n158a13125202pdf001.pdf\&anuncioPdf=ERROR_pub li_pdf

34. Cruz Pierard SM, Zurita Baquero JF. Composición corporal de los docentes a tiempo completo de la Facultad de Enfermería de la Pontificia Universidad Católica del Ecuador en relación con su ingesta calórica $\mathrm{y}$ de macronutrientes [tesis en Internet]. [Quito]: Pontificia Universidad Católica del Ecuador; 2015 [citado 26 de noviembre 2016]. Disponible en: http://repositorio.puce.edu.ec/handle/22000/9238

35. Sakraida J. Modelo de Promoción de la Salud. Nola J Pender. En: Marriner-Tomey A, Raile Alligood M. Modelos y teorías en Enfermería. Madrid: Elsevier 2011.

36. Gómez Campo VM, Celis Giraldo JE. Docencia, estatus, distinción y remuneración. Nómadas (Col) [Internet]. 2007;(27):98-109. Recuperado de: http://www.redalyc.org/articulo.oa?id=10511659500 9
37. Colombia. Decreto 1279/2002, de 19 de junio, de Presidencia de la República, por el cual se establece el régimen salarial y prestacional de los docentes de las Universidades Estatales.

38. Solano Benavides E, Navarro G. Remuneración salarial eficiente en la educación superior en Colombia. Economía, XXXVII. 2012;33:43-63.

39. Vélez-Laguado P, Mayorga-Ayala LD. Relación de los factores de riesgo psicosociales y el síndrome de burnout en docentes de planta de la Universidad Francisco de Paula Santander en el periodo del 20112012. Cienc y Cuid. 2013;85-95.

40. Verdugo Lucero JC, Guzmán Muñiz J, Moy López NA, Meda Lara RM, González Pérez OP. Factores que influyen en la calidad de vida de profesores universitarios. Centro [Internet]. 2007;18:27-36. Disponible http://www.redalyc.org/pdf/291/29118103.pdf

41. Rojas B ML, Zapata H JA, Grisales R H. Sindrome de burnout y satisfacción laboral en docentes de una institución de educación superior, Medellin, 2008. Rev la Fac Nac Salud Pública. 2009;27(2):198-210.

42. Giraldo Osorio A, Toro Rosero MY, Macías Ladino AM, Valencia Garcés CA, Palacio Rodríguez S. La promoción de la salud como estrategia para el fomento de estilos de vida saludables. Hacia la Promoción la Salud. 2010;15(1):128-43.

43. Restrepo Ayala NC, Colorado Vargas GO, Cabrera Arana GA. Desgaste Emocional en Docentes Oficiales de Medellín, Colombia, 2005. Rev Salud Pública. 2006;8(1):63-73.

44. Perea-Baena JM, Sánchez-Gil LM. Estrategias de afrontamiento en enfermeras de salud mental y satisfacción laboral. Index Enferm [Internet]. 2008 Sep [citado 2017 Dic 25]; 17(3): 164-168. Disponible en: http://scielo.isciii.es/scielo.php?script=sci_arttext\&pid $=$ S1132-12962008000300003\&lng=es 